\title{
Publisher Correction: Promoting nitrogen electroreduction to ammonia with bismuth nanocrystals and potassium cations in water
}

Yu-Chen Hao, Yu Guo, Li-Wei Chen, Miao Shu, Xin-Yu Wang, Tong-An Bu, Wen-Yan Gao, Nan Zhang, Xin Su, Xiao Feng iD, Jun-Wen Zhou, Bo Wang, Chang-Wen Hu, An-Xiang Yin (D), Rui Si, Ya-Wen Zhang (D) and Chun-Hua Yan

Correction to: Nature Catalysis https://doi.org/10.1038/s41929-019-0241-7, published online 4 March 2019.

In the version of this Article originally published, all labels for the orange, blue and red trends in Fig. 3b read 'BiNCs', but the orange labels should have read 'BiNPs', and the blue 'BiBPs'. This has now been corrected.

Published online: 8 March 2019

https://doi.org/10.1038/s41929-019-0267-x 